\title{
A CASE OF TUBERCULOUS PERICARDITIS
}

\author{
BY \\ A. M. BARRETT AND LESLIE COLE \\ From the Department of Pathology, University of Cambridge, and Addenbrooke's Hospital \\ Received October 21, 1944 \\ Uncomplicated tuberculous pericarditis is not common, and this case is described because \\ it gives a good picture of the march of the disease both clinically and pathologically.
}

\section{DesCription OF CASE}

A man, aged 22, who worked as a shop assistant, was admitted to Addenbrooke's Hospital on March 11, 1943, with the following history. About mid-February he first began to feel generally out of sorts and over the space of a few days developed a dry, irritating cough which kept him awake at night, a dull pain over the præcordium, nausea, weakness, and slight breathlessness on exertion. These symptoms persisted until his admission.

His previous health had been good and the only illness he remembered was an attack of " pleurodynia " at the age of 18 . His family history was healthy with no tuberculosis.

On admission the heart was greatly enlarged both to left and right, the apex beat could not be seen or felt, the sounds were faint and tic-tac in quality, the pulse small, regular, and rapid (110), the respiratory rate 22 , the temperature intermittent and rising to 100 or 101 , and the blood pressure $110 / 80$.

The móst arresting physical sign was a loud rub audible over the whole pericardium but most marked at the base.

The cardiogram showed low voltage curves with inversion of $T$ in leads I, II, IVR, and IVF (Fig. 1) and X-ray showed the typical shadow of a large pericardial effusion (m.t.d. $17 \mathrm{~cm}$.; see Fig. 2). The sedimentation rate (Westergren) was $60 \mathrm{~mm}$. in one hour.

Progress. Except for a period of two months in September and October when he rested at home, he remained under observation in hospital until his death on December 9.

The progress of the disease appeared to fall into three clinical phases.

(1) The phase of increasing effusion leading to congestive failure (4 months).

(2) The phase of decreasing effusion with temporary recovery (3 months).

(3) The phase of increasing thickening and fibrosis of the pericardium and congestive failure ending in death ( 3 months).

Phase 1. During this phase the rub disappeared, there was a progressive increase in the size of the effusion and the temperature fell gradually to normal in six weeks. In the first few weeks the blood pressure fell to an average of $90 / 65$ at which level it remained for about three months and the veins of the neck became distended and remained so. This phase culminated in an attack of congestive failure with a large pleural effusion at the right base, some ascites and slight general œdema.

Phase 2. Following recovery from the congestive failure (Fig. 3)
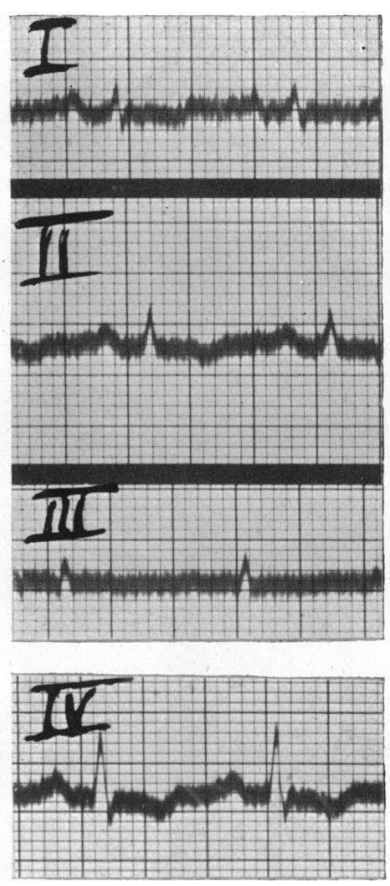

Fig. 1.-Cardiogram (25/4/43) showing P-R 0.16 sec. and inversion of $T$ waves.

the pericardial effusion began to subside gradually and there was marked general improvement with a steady rise of blood pressure to an average level of 110/80 and lessening venous distension. By the end of July his resting pulse rate was 80-100, and he appeared to 


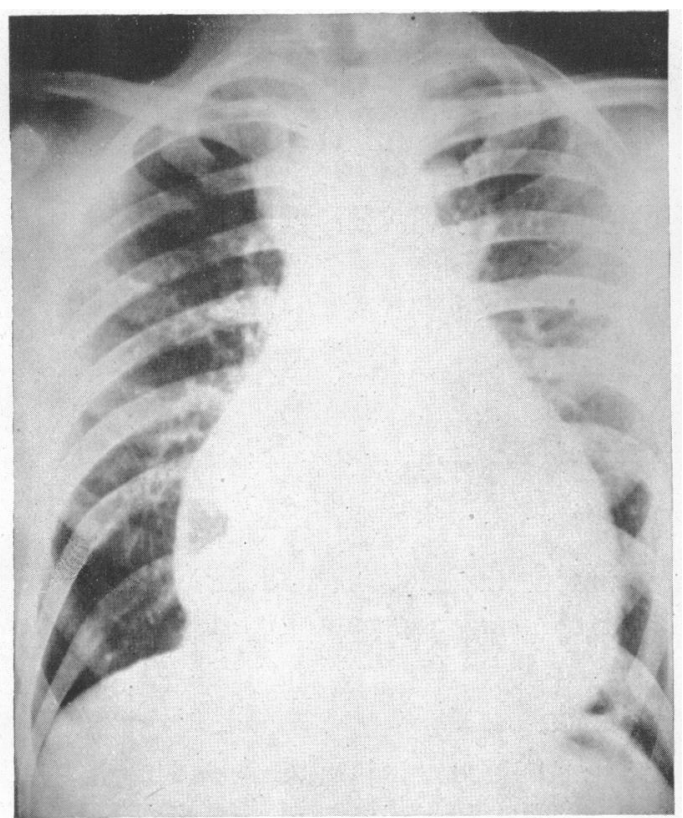

FIG. 2.-Radiogram of the heart (2/4/43) showing rounded dilatation of the pericardial sac.

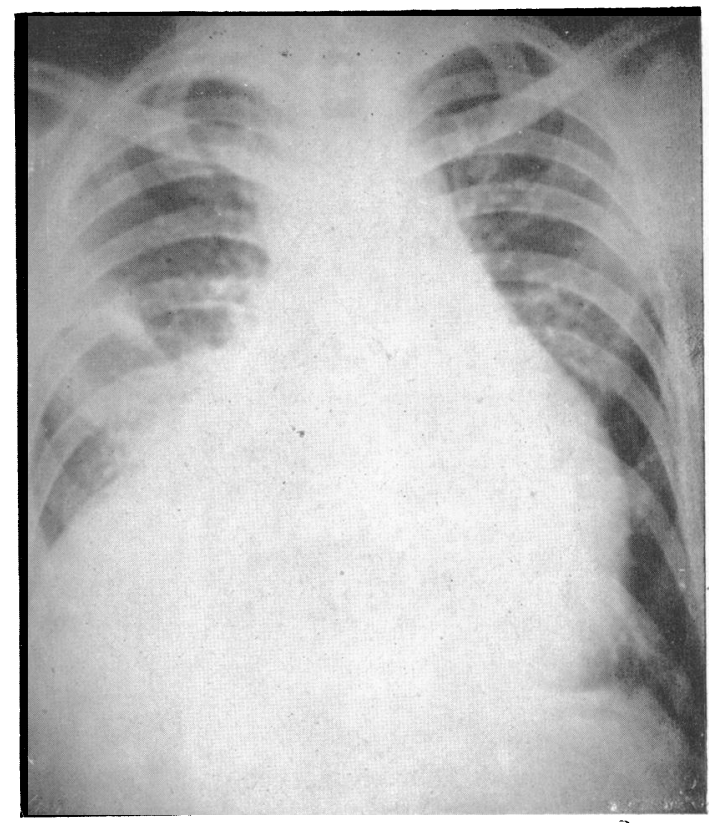

FIG. 3.-Radiogram of the heart (8/7/43) after recovery from an attack of congestive failure with right basal effusion.

have recovered some measure of compensation. His sedimentation was then $12 \mathrm{~mm}$. His condition then appeared to remain stationary and had not changed when he was readmitted for further observation in October.

Phase 3. The diminution in size of the area of cardiac dullness with increasing irregularity of outline by X-rays suggested progressive thickening of the pericardium (Fig. 4). In November, steady deterioration began. The pulse rate gradually rose from an average of 80 , the blood pressure steadily fell from an average of $110 / 85$, the pulse pressure also fell, and the venous congestion again became more marked and pulsus paradoxus very pronounced. Congestive failure with gross œdema

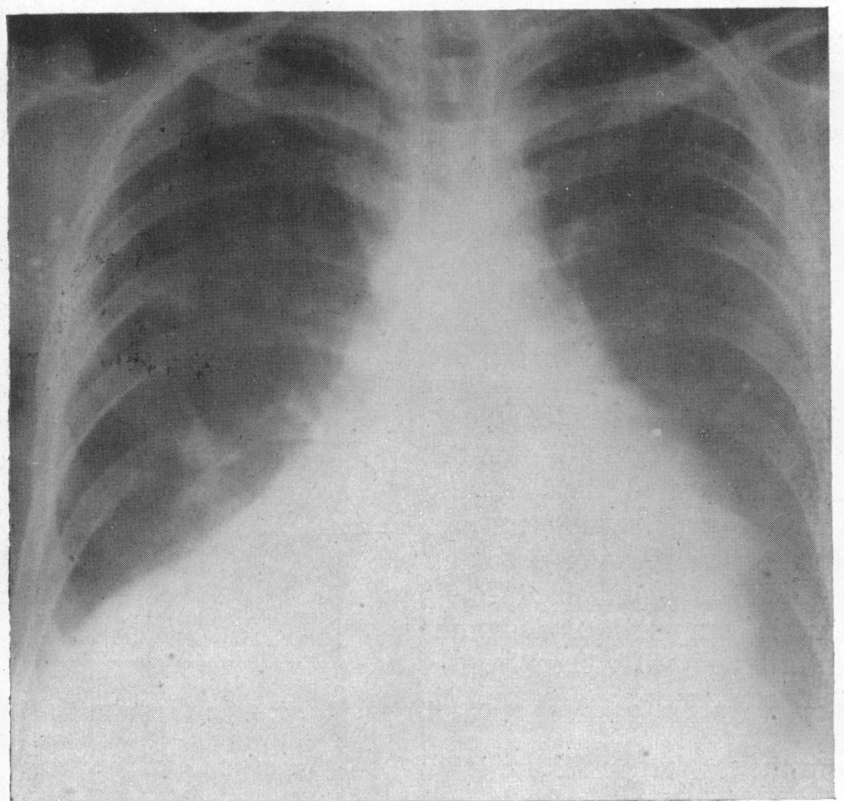

FIG. 4.-Radiogram of the heart $(27 / 10 / 43)$ showing the pericardial sac diminished in size and irregular in outline.

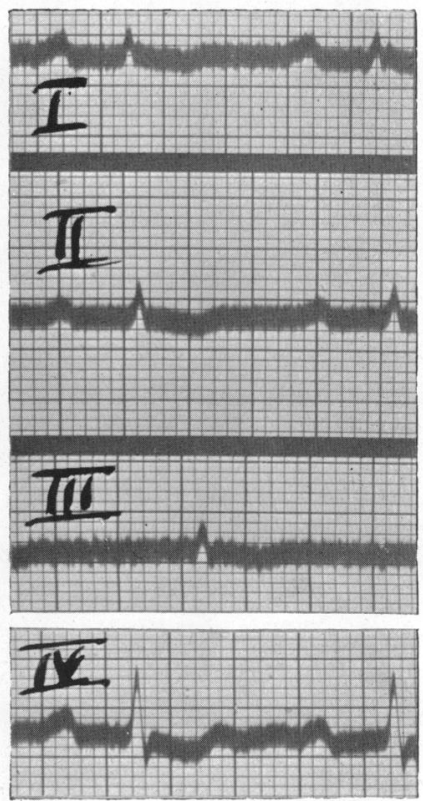

FIG. 5.-Cardiogram (28/10/43), latent heart block with P-R $0 \cdot 24$ sec. 
gradually supervened and he died on December 9. During the last two months the sedimentation rate was below $6 \mathrm{~mm}$. The cardiogram at the end of October showed still lower voltage curves with T III also inverted and the P-R interval, which had been previously normal, prolonged to $0.24 \mathrm{sec}$. (Fig. 5).

The leucocyte count $(27 / 5 / 43)$ was 9,800 cells per c.mm. The differential count was normal.

Pericardial fiuid. This was yellowish and slightly opalescent with a fine feathery clot. A few small lymphocytes and some fibrin were present but no red cells. Cultures were sterile. A guinea-pig inoculated on April 28 appeared to remain well until October but died on October 8. The cause of death was very extensive, rather hyperplastic tubercle involving glands, liver, spleen, and lungs. Tubercle bacilli were found in stained films. A Mantoux test $(1 / 11 / 43 ;$ human, $1: 10,000)$ was positive.

Treatment. 300 c.c. of fluid were aspirated slowly from the pericardium on April 27. This did not appear to have any beneficial effect on either the general or local condition, nor did it cause any change immediate or remote on the very low blood pressure. The general condition was worse during the succeeding few days, and for this reason it was not repeated. Suzman (1943) noted the absence of any effect on the blood pressure in a similar case.

During the first attack of congestive failure, ammonium chloride, 30 grains, followed by mersalyl, 1 c.c. and later 2 c.c. intramuscularly, were given with only moderate response. The fourth injection was followed in three minutes by severe collapse in which the patient nearly died, after which its use was abandoned. Digoxin was given at various times, but only appeared to have a beneficial effect during the first attack of congestive failure. Aspiration of the pleural effusion caused marked and sustained improvement.

\section{Cuinical Comment}

The diagnosis of tuberculous pericarditis was made in the early stages on the clinical findings, but was only confirmed by guinea-pig inoculation; it is noteworthy that the guineapig appeared to maintain health for five months after the inoculation, an unusually long period. Constitutional symptoms in the patient were notably slight throughout the illness. The temperature settled during the first six weeks and did not rise again, the appetite remained fairly good, and there was no abnormal sweating. After the first few weeks the sedimentation rate was never higher than $12 \mathrm{~mm}$. in one hour. Apart from the two attacks of congestive failure, symptoms were very slight. During the second phase there was considerable improvement with a rise of blood pressure and diminution of venous congestion, and for about three months there did not appear to be any deterioration. Reviewing the whole course of the disease, it seems probable that this apparent improvement was illusory and occurred because the relief afforded by the absorption of excessive pericardial fluid had not yet been offset by the gradual thickening of the visceral pericardium with its progressive hampering effect on the action of the heart. As this process became more advanced, terminal failure gradually set in.

\section{POST-MORTEM EXAMINATION}

A deeply cyanosed well-developed young 'man with œdema and engorgement of veins in neck and elsewhere. Great excess (740 c.c.) of cloudy greenish-lemon fluid in much enlarged pericardial sac, which has a thick deposit of gelatinous yellowish fibrin on its inner surface, particularly over the right auricle and on the adjacent parietal pericardium (Fig. 6). Pericardium everywhere greatly thickened $(0 \cdot 2-0 \cdot 3 \mathrm{~cm}$. thick) and tough, grey to pinkish-grey in colour where it is neither covered by fibrinous exudate nor beset with greyish-white granulomatous plaques of which there are many. No adhesions between parietal and visceral pericardium except slightly at base. No constriction, not even of slight degree, of superior or inferior venæ cavæ nor of the main pulmonary vessels. Rather small heart, its ventricles almost empty and its auricles containing less than 50 c.c. blood and clot in all. Myocardium brown or brownish-red and apparently normal (right ventricle $0.6-0.7 \mathrm{~cm}$. thick, left ventricle $1.5 \mathrm{~cm}$. thick). Heart valves normal. Conspicuous hypertrophy of the wall of the inferior vena cava, which immediately below the liver appears about twice as thick as normal and is sufficiently rigid to prevent the collapse of the vessel after it has been opened. Only slight 
Fig. 6.-Photograph of the heart showing a thickened and distended pericardium beset with granulomatous plaques.

atheroma of aorta, its abdominal part being rather narrower than the average but within normal limits. No excessive atheroma of pulmonary arteries. Non-specific inflammatory changes only in several moderately enlarged lymph glands on the outer surface of the pericardium near the hilum of the left lung. Ill-defined firm greyish areas suggesting granulomatous tuberculosis in several lymph glands on the left posterior aspect of the pericardium. Caseous tuberculosis of one small mediastinal lymph gland near the left bronchus, between the main left pulmonary artery and the arch of the aorta; this gland was about $1.5 \mathrm{~cm}$. from the pericardium at its nearest point, and its soft crumbling centre surrounded by a dense zone of fibrosis. Small $(0 \cdot 2 \mathrm{~cm}$. diam.) calcareous nodule surrounded by fibrous tissue in upper lobe of left lung near apex. Both lungs partially collapsed by pleural effusions and slightly congested. Clear yellowish fluid in serous cavities: right pleura 1870 c.c., left pleura 920 c.c., peritoneum 2640 c.c. Mesenteric lymph glands rather large but normal in appearance. Changes in the other organs were those of chronic venous congestion. Heart not weighed because preserved with pericardium for mounting.

Microscopic. Heart. Chronic tuberculous pericarditis with much increase of fibrous tissue. No definite lesions in myocardium. Lymph glands (from posterior surface of peri- 
cardium). Typical granulomatous tuberculosis with abundant giant cells but no caseation. Inferior vena cava. Conspicuous thickening of wall. Mesenteric lymph gland. Very wide but almost empty sinuses and atrophy of lymphoid tissue; no tuberculosis.

The changes in the other organs were those of chronic venous congestion.

Bacteriological. By guinea-pig inoculation with pericardial fluid tubercle bacilli of eugonic human type were demonstrated.

\section{The Mechanism of Impairment of CARdiac Function}

During the necropsy a somewhat crude attempt was made to determine whether the pericardial effusion itself at the time of death was likely to have caused any serious impairment of cardiac function.

Presumably such impairment would be mainly due to limitation of the intake of blood into the auricles during diastole. If $\mathrm{V}(\mathrm{e})=$ the volume of the effusion, $\mathrm{V}(\mathrm{b})=$ the total volume of blood that enters and is expelled from the heart at each beat, and $V(h)=$ the volume of the substance of the heart itself, then impairment of cardiac function due to an effusion will not be expected unless $\mathrm{V}(\mathrm{e})+\mathrm{V}(\mathrm{b})+\mathrm{V}(\mathrm{h})$ is sufficiently great to stretch the parietal pericardium so that before the end of diastole the pressure inside the pericardium (and hence in the auricles) becomes equal to that in the venæ cavæ. $V(b)$ will then be reduced and the function of the heart impaired. We attempted at necropsy to discover whether this critical volume had been attained by measuring the relationship between known increases in the total volume of the pericardial contents and the intra-pericardial pressure.

When the thorax and abdomen had been opened, and before any further dissection, the superior and inferior venæ cavæ were ligatured immediately above and below the intact pericardium. This was done in order to prevent alterations in the volume of the pericardial contents due to blood being squeezed out of the heart during the subsequent manipulations. A glass funnel was attached to one end of a length of rubber tubing and a blood transfusion " taking" needle to the other end. The needle was then inserted into the pericardium (through the diaphragm, to minimize leakage) and 35 c.c. pericardial fluid was allowed to escape through the tube into a bottle. This sample was reserved for examination, but the original volume of the effusion was restored by pouring 35 c.c. water back through the funnel into the pericardial sac. The funnel was now held in such a position that a mark on its stem was $10 \mathrm{~cm}$. above the pericardium and water was poured in until the level remained stationary at the mark. The volume of water that had been added was noted, and the funnel was then raised until the mark was $20 \mathrm{~cm}$. above the pericardium. The process was then repeated; and similarly with the level at $30 \mathrm{~cm}$., and $50 \mathrm{~cm}$. The same measurements were made on another subject whose heart and pericardium appeared normal. The results were as follows:
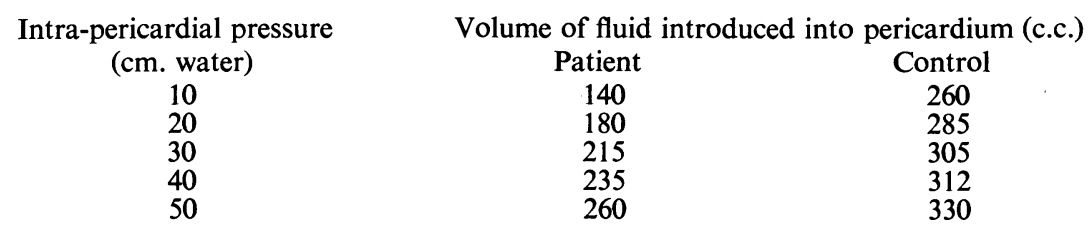

Strictly, in comparing these measurements, allowance should be made for the volume of blood and clot present in the chambers of each heart, but it was not found possible to measure this volume accurately without spoiling the heart as a museum specimen, and this we were loath to do. However, the volume of the contents was relatively small (less than 50 c.c.) in each of the hearts and there was no great inequality in the amounts.

These results suggest that an effective venous pressure of $10 \mathrm{~cm}$. water should have enabled the heart to deal with at least 140 c.c. blood at each beat if its action were hampered only by the mechanical effects of the effusion. The average systolic output of normal adults at rest 
is between 60 and 70 c.c. (Grollman, 1932), but this figure is the output of one ventricle only: the total output of the heart is $120-140$ c.c. per beat. Thus it seems that in spite of the effusion the patient should have been able to maintain a normal cardiac output while resting, provided that his effective venous pressure was not less than $10 \mathrm{~cm}$. water. His actual venous pressure was not measured, but the conspicuous venous engorgement observed during life and the thickening of the wall of the inferior vena cava seen at necropsy suggest that the pressure was probably greater than this, and hence that the impairment of cardiac function about the time of death was not due to the mechanical effects of the effusion.

Of other possible causes of impairment of cardiac function, the greatly increased thickness and rigidity of the visceral pericardium appears the most likely. There was little histological evidence of myocardial damage although this had been indicated by the prolonged $\mathrm{P}-\mathrm{R}$ interval of the cardiogram. Constriction of the vessels entering the heart was excluded.

\section{SUMMARY}

This patient's initial tuberculous infection was situated near the apex of the left lung and was very small. The infection apparently spread from the lung to a mediastinal lymph gland between the left bronchus and the pericardium, and from this gland to the pericardiumthough no definite track of infection between the gland and the pericardium was in fact found at necropsy. Tuberculous pericarditis with effusion followed, and death was due to congestive heart failure. There was no evidence of tuberculosis elsewhere in the body. Evidence is given which suggests that at the time of death the pericardial effusion played a relatively small part in limiting cardiac efficiency, and it is suggested that increased thickness of the visceral pericardium with loss of its pliability was the chief cause.

We wish to thank Dr. J. F. Gaskell for permission to investigate and publish this case, Dr. C. H. Whittle for some of the pathological investigations, and Dr. Ff. Roberts for the X-ray examinations.

\section{REFERENCES}

Grollman, A. (1932). The Cardiac Output of Man in Health and Disease, London. Suzman, S. Brit. Heart J., 5, 19. 